\title{
Interfacial thermocapillary vortical flow for microfluidic mixing
}

Ramanathan Muruganathan, Yi Zhang and Thomas M. Fischer ${ }^{1}$

\author{
Department of Chemistry and Biochemistry, Florida State University, \\ Tallahassee, Florida, USA
}

\section{Supporting information}

\section{S1. Experimental}

S1.1) Set-up:

Our setup consists of a homebuilt Langmuir trough with a fluorescence microscope that works with a 100x water immersion objective, numerical aperture 1.0, built into the bottom of the temperature controlled film balance. The same objective is used to focus an IR-laser beam $(\lambda=1064 \mathrm{~nm}, P=10 \mathrm{~mW}-4 \mathrm{~W})$ onto the central imaging region of the monolayer. The main purpose of this laser is to locally heat the monolayer by the absorption of water (absorption coefficient of water at $\lambda=1064 \mathrm{~nm}: \alpha \approx 0.1 \mathrm{~cm}^{-1}$ ). Quantifying the laser heating:

The applied laser power $(10 \mathrm{~mW}-4 \mathrm{~W})$ causes an observable local heating. This can melt the low-temperature phase in a locally defined region $(10 \mu \mathrm{m} \leq \mathrm{r} \leq 100 \mu \mathrm{m})$. In the beam waist of the laser, a fraction of $10^{-6}$ of the laser power $P$ is converted into heat. Assuming this heat is dissipated into the subphase by heat conduction only, the rise in temperature within the focus is estimated to be $\Delta T=\alpha \frac{3 P}{4 \pi \kappa_{w}}$. Here, $\kappa_{\mathrm{w}} \approx 0.6 \mathrm{~W} / \mathrm{m} . \mathrm{K}$, is

\footnotetext{
${ }^{1}$ To whom correspondence should be addressed, E-mail: tfischer@chem.fsu.edu
} 
the heat conductivity of water therefore by applying $1 \mathrm{~W}$ laser power creates the local temperature difference of $\Delta \mathrm{T}, 5 \mathrm{~K}$.

\section{S1.2) Lipid monolayer}

L- $\alpha$-dipalmitoylphosphatidyl-choline (DPPC) and 1,2-dipalmitoyl-sn-glycero-3\{phosphor-L-serine\}(sodium salt) (DPPS) were purchased from Avanti Polar-lipid Inc., and used without further purification (99+\% purity). NBD-PC and NBD-PS were used as a fluorescent marker respectively for DPPC and DPPS. The experiments with the lipid monolayers were performed at the room temperature $\left(19-21^{\circ} \mathrm{C}\right)$.

Folds in the monolayer:

It is known that a Langmuir monolayer can fold into the sub phase at appropriate conditions $^{1,2}$. In a recent paper $^{3}$ we showed the kinetics of irreversible fold speed in a mixed phopholipid monolayer. Here we use 1:1 DPPC-DPPS mixed phopholipid monolayer to create folds. $10 \mathrm{mM}$ lipid mixture was dissolved in chloroform and mixed with $0.2 \%$ of fluorescent dye (1:1 NBD-PC and NBD-PS) then spread on top of the water surface. Compressing this monolayer below $70 \AA^{2}$ per molecule will form folds in the

monolayers. In this study, the formed monolayer was slowly compressed to $45 \AA^{2}$ per molecule and left at high pressure for about 15minutes to ensure the formation of higher percentage of folds in the monolayer. . Later the mechanical barriers were opened to an area per molecule of $100 \pm 10 \AA^{2}$ to form the LE phase at which the experiments are performed. Figure 1-right shows the fluorescence image of the folds in the monolayer 


\section{S2. Derivation of equation (1)}

Here we calculate the stream function, velocity and vorticity of a monolayer subject to a torque located at the air/water surface. Since Reynolds number is much smaller than one it is appropriate to use equations of creeping flow. For the range of concentration used in our experiments the surface shear viscosity is negligible. We start with the cylindrical symmetric stream function

$$
\Psi(r, \vartheta, z)=\Omega R^{2} \int_{0}^{\infty} d s s^{-1} a(s) J_{0}(s r / R) e^{-k s z / R}
$$

where $a(s)$ is a function of $s$ to be determined, $J_{n}(s r / R)$ is the Bessel function of order $\mathrm{n}$ and argument $s r / R$. $\Omega$ is a prefactor of the typical order of the vorticity and R is the lateral length scale of the vortex. The stream function solves the Laplace equation $\nabla^{2} \psi=0$ and as a consequence the velocity $\mathbf{u}=\nabla \times\left(\mathbf{e}_{z} \Psi\right)$ fulfills the Stokes equation and the incompressibility condition $\nabla \cdot \mathbf{u}=0$ in the bulk. Using $\omega=\nabla \times \mathbf{u}$ we find:

$$
\begin{gathered}
u_{r}(r, z)=0 \\
u_{\vartheta}(r, z)=-\Omega R \int_{0}^{\infty} d s a(s) J_{1}(s r / R) e^{-s z / R} \\
u_{z}(r, \vartheta, z)=0 \\
\omega(r, z)=\Omega \int_{0}^{\infty} d s s a(s) J_{0}(s r / R) e^{-s z / R}
\end{gathered}
$$

We obtain the inversion of the last equation in (S2) when setting $\mathrm{z}=0$ multiplying the vorticity $\omega(\mathrm{r}, 0)$ with $r J_{0}(s r / R)$ and integrating over $\mathrm{r}$.

$$
a(s)=\int_{0}^{\infty} \frac{d r}{R} \frac{\omega(r, 0)}{\Omega} \frac{r}{R} J_{0}(s r / R)
$$


The fluid motion of the liquid expanded and gaseous phase at the air/water interface obeys the $2 \mathrm{~d}-$ Stokes equation. Since the viscous stresses are small as compared to Marangoni forces the surface is essentially incompressibility:

$$
\begin{gathered}
\left.-\nabla_{s} \pi_{m}+\eta \frac{\partial \mathbf{u}}{\partial z}\right)_{s}-f_{\mathbf{s}} \mathbf{e}_{\vartheta}=0 \quad \text { for } \quad z=0 \\
\nabla_{s} \cdot \mathbf{u}_{\vartheta}=0 \quad \text { for } \quad z=0
\end{gathered}
$$

Here $\pi_{\mathrm{m}}$ is the surface pressure of the monolayer, $\nabla_{\mathrm{s}}$ the surface gradient, the second term is the viscous drag force from the subphase and the last term describes the force density including all surface force sources other than those arising due to surface pressure gradients or bulk fluid traction. The surface pressure gradient vanishes for symmetry reasons. The radial torque density acting at the radius $r$ is given by

$$
\tau(r)=-2 \pi r^{2} f_{s}
$$

And insertion of equations (S2) - (S4) into (S5) results in:

$$
\begin{aligned}
\tau(r) & =-2 \eta \pi r^{2} \frac{d}{d r} \int_{0}^{\infty} d r^{\prime} \frac{r^{\prime} \omega\left(r^{\prime}\right)}{R} \int_{0}^{\infty} d s J_{0}\left(s \frac{r}{R}\right) J_{0}\left(s \frac{r^{\prime}}{R}\right) \\
& =-4 \eta r^{2} \frac{d}{d r} \int_{0}^{\infty} d r^{\prime} \omega\left(r^{\prime}\right) \frac{r^{\prime}}{r+r^{\prime}} K\left(\frac{2 \sqrt{r r^{\prime}}}{r+r^{\prime}}\right)
\end{aligned}
$$

where $K(k)$ is the complete elliptic integrals of the first kind ${ }^{1}$. Equation (S5) is the same as equation (1).

Following the same kind of analysis we can express the vorticity at a certain depth $z$ as

$$
\omega(r, z)=\int_{0}^{\infty} \frac{d r^{\prime}}{R} \frac{r^{\prime} \omega\left(r^{\prime}, 0\right)}{R} \int_{0}^{\infty} d s J_{0}\left(s \frac{r}{R}\right) J_{0}\left(s \frac{r^{\prime}}{R}\right) e^{-s z / R}
$$

The integral over $s$ in (S6) can be performed if $r=0$ and we obtain 


$$
\omega(0, z)=\int_{0}^{\infty} d r^{\prime} \frac{z r^{\prime}}{\left(z^{2}+r^{\prime 2}\right)^{3 / 2}} \omega\left(r^{\prime}, 0\right)
$$

It is easy to see from (S7) that the penetration depth of the vorticity is of the same order than its lateral extension at the surface.

\section{REFERENCE:}

${ }^{1}$ Gopal, A.; Lee, KYC. J. Phys. Chem. B. 2001, 105, 10348.

${ }^{2}$ Piknova, B.; Schram, V.; Hall, S. B. Current Opinion in Structural Biology, 2002, 12 , 487.

${ }^{3}$ Zhang, Y. and Fischer, Th. M. J. Phys. Chem. B. 2005, 109, 3442 